\title{
Research on the New Image De-Noising Methodology Based on Neural Network and HMM-Hidden Markov Models
}

\author{
Wenzhun Huang ${ }^{1, a}$ and Xinxin Xie ${ }^{1, b}$ \\ ${ }^{1}$ School of Information Engineering, Xijing University, Xi'an 710123, China

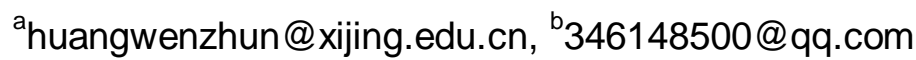

Keywords: De-noising; Methodology; Neural network; HMM; Markov models

\begin{abstract}
In this paper, we conduct research on the new image de-noising methodology based on the neural network and HMM-hidden Markov models. HMM is a double stochastic process, one of which is a Markov chain, this is basic random process as it describes the state of the shift and another random process description statistics corresponding relations between the state and the observation. We apply the method into the image de-noising application that will enhance the general performance with the better accuracy. In the future, we will integrate the simulation steps to verify the effectiveness.
\end{abstract}

\section{Introduction}

It is the purpose of image enhancement and recovery in order to improve the quality of images, such as, remove noise, enhance image clarity, etc. Image enhancement is not consider images with reason, highlight a part of the image are interested in. Such as, to strengthen the image of the high frequency component as can make the objects in the image contour is clear, the details; Such as strengthen the low-frequency component can reduce the image noise. Image restoration for reasons in the quality of the images have a certain understanding, generally speaking should be established according to the mass reduction process "drop qualitative model", again with some kind of filtering method, restore or rebuild the original image [1-3].

With traditional frequency domain denoising based on image information in the frequency domain space characterized by the combination of different frequencies, which the basic noise of the image corresponding to the high frequency part of the Fourier spectrum by inhibiting or eliminate the high frequency part of image denoising. The traditional image denoising methods have only airspace and frequency domain localization analysis ability, thus to suppress image noise at the same time, also can damage the image edge details, after denoising image blur.

In view of the existing local fuzzy measurement method is easy to smooth texture clear regional mistakenly identified into fuzzy region, the deficiency of the study use all singular value of singular value vector fuzzy detection is proposed. Because the texture smooth region and fuzzy region has a large number of large scale information and the fewer small scale information, without considering the fuzzy after large scale change of information is necessary to smooth texture clear error for fuzzy region area. Neural network is a kind of application is similar to the structure of the brain synapses connection for information processing, the mathematical model can well reflect the fuzzy awareness. BP network is a multilayer neural network mapping that can approximate any the complex nonlinear relations, fully uses the error back-propagation learning algorithm to the optimal parameters, such as cumulative values which has the advantages of extensive adaptability and effectiveness [4-5].

HMM is a double stochastic process, one of which is a Markov chain, this is basic random process as it describes the state of the shift and another random process description statistics corresponding relations between the state and the observation. So, stand in the angle of the observer, that only with observations, unlike Markov chain in observation value and status. So we can't see the status directly, but through a random process to the existence of the state of perception and core features. Traditional Markov chain to easy to distinguish the discrete state that has a very good prediction effect. However, when the state space is approximate continuous value, the predicted result may create a status as the data change, leap. In addition, when the state shift, may not be able to determine the Markov chain in 
the next step what is the only state, i.e., a description of the state can't exactly give. The essence of these problems is the process of Markov chain sequence is uncertain, main show is the state of the object of study can divide clearly that will be discussed furtherly.
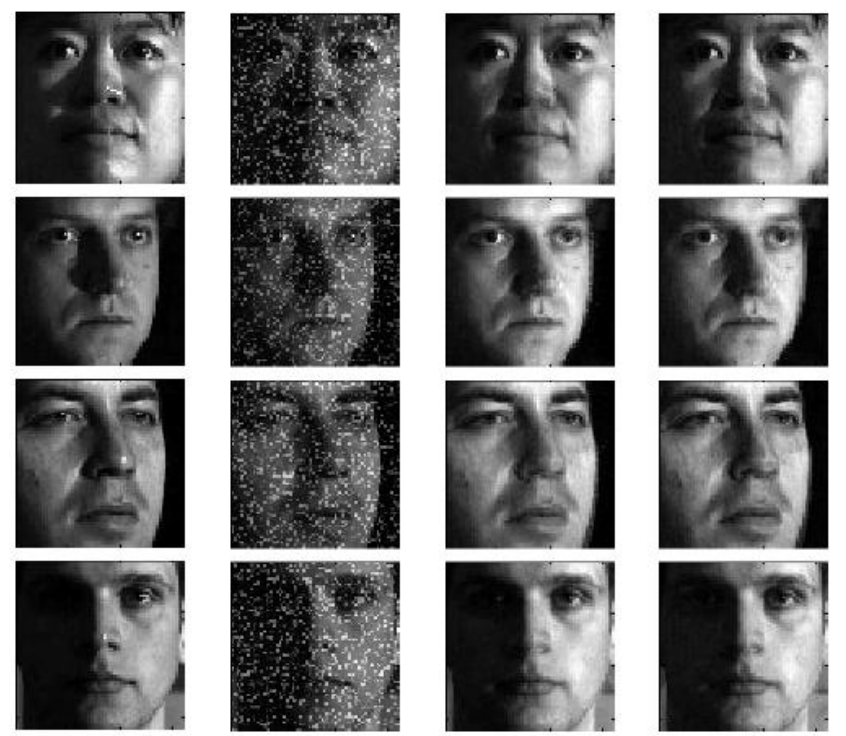

Figure 1. The Image De-noising with the Appications on the Face Database

In this paper, we conduct research on the new image de-noising methodology based on the neural network and HMM-hidden Markov models. Adaptive estimation method to the sampling interval integer times after the time delay estimation using the algorithm estimate the decimal part, and then improve the estimation precision. In the later sections, we will discuss in detail.

\section{Description of the Algorithm}

The Image Statistical Properties. Currently used methods for statistical model parameter estimation are maximum likelihood estimation, with maximum a posteriori probability estimation, the maximum entropy estimation and maximum expected algorithm, etc.

In image processing, is often a priori information of probability density function is unknown, but it is known to the relevant constraints, such as a range of points or the local statistical distribution of the image and use the information in the solution of a priori information as can be converted to a given constraint conditions prior information of the maximum entropy estimation. If a priori information known probability distribution function of the parameters, the maximum entropy estimation of prior information model, the uniform distribution on if a priori information model parameters of the mean and variance of the unknown. Feature extraction is the key to the subsequent image processing steps as it will directly affect the image retrieval, matching, and the result of segmentation. So in the past few decades, the selection of image characteristics has always been a key research by scholars at home and abroad, and the texture is essential in all the characteristics of one of the important characteristics, the study of it never stopped, and made great progress. Expected algorithm is mainly composed of two steps, the maximum expected step and the step, in the process of iterative calculation, increase the likelihood function value of the complete data and make its convergence to a local or global optimal solution and obtain maximum likelihood estimates of the model parameters as follows [6].

$$
\theta^{(n+1)}=\arg \max \Theta\left(\theta \mid \theta^{(n)}\right)
$$

Expectation maximum algorithm of the state and the parameter estimation that coupled together, namely in the parameter estimates that need to know the state estimate, and need to know the estimate of parameters in state estimation, makes the estimate of parameter estimate and state influence each 
other, if the parameter estimation to get the basic local optimal solution, the state will not be able to accurately approximate completely the statistical distribution of the data set as follows.

$$
\Theta\left(\theta^{(n+1)} \mid \theta^{(n)}, y\right)>\Theta\left(\theta^{(n)} \mid \theta^{(n)}, y\right)
$$

For reflecting the continuous characteristics of wavelet coefficient of basic amplitude, the scale of general wavelet coefficients between relationships with first-order Markov state transition probability to describe, complete the distribution of the dataset described using hidden Markov model. But the Gaussian mixture model and static image using the Gaussian mixture model with different nature, the algorithm for segmentation of video will be divided into interested targets and noise two small objects or incomplete data sets, are described using the Gaussian mixture model, each frame of incomplete data sets statistical distribution determined by the product of the two. Parameters of the final solution is closely related to the initial conditions, while if the parameter improper choice of initial condition, eventually lead to fall into local extremum, it gives accurate statistical model parameters estimated causing very bad influence that will affect the subsequent image processing.

The Neural Network. The neural network structure optimization problem has always been a hot spot of research at home and abroad. Network on the number of input nodes and output node number can be determined by the actual problem, key points and difficulties in optimizing the structure of the network is the design of the hidden layer structure as the specific refers to the number of hidden layer and the number of hidden layer neurons. Determine the structure of the hidden layer to a great extent, decides the memory capacity of the network, generalization ability, the training speed and the quality of the output response. The figure two demonstrates the principles [7].

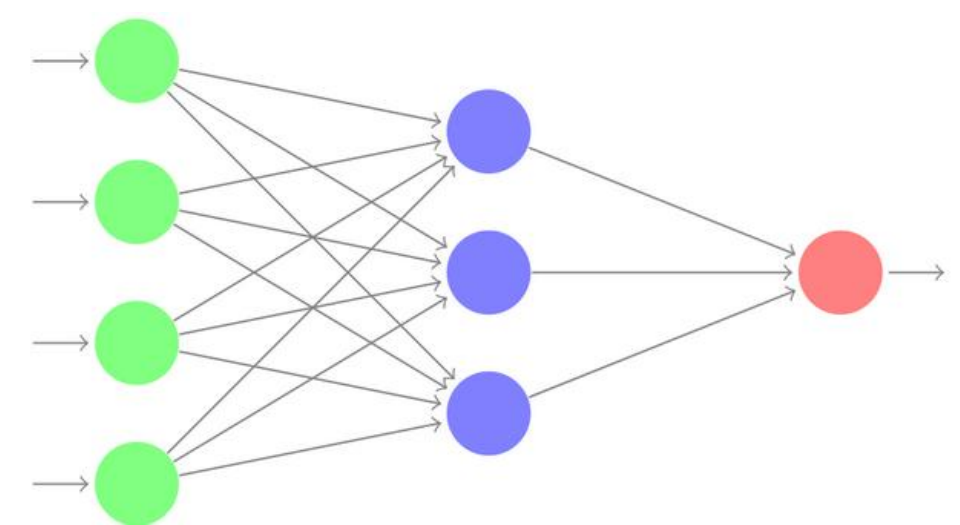

Figure 2. The Neural Network Model

Combined with the network to the input and output has good nonlinear mapping ability, it is to the network before all the optimal network, complete the mapping function of neural network in this paper, the convolution in the last layer down sampling with the original output layer between the add a layer of a better classification performance of radial hidden layer, and increase the original last layer down sampling number of neurons, to further improve the deep learning network using the ability of the self-learning feature classification with the objective of minimizing formula 3.

$$
E(Z)=\frac{1}{2 K} \sum_{k=1}^{K} \sum_{j=1}^{J}\left(d_{k}^{j}-y_{k}^{j}(Z)\right)^{2}
$$

Multilayer feedforward neural network error function constitute a number of general independent equations of nonlinear equations and the network and the number of unknown variables should be equal export have cross connection layer of multilayer feedforward neural network structure equation. Based on cross connection layer of multilayer feedforward neural network, through the improvement of connection mode, abandon the traditional neural network structure based on the perfect connection mode, help to reduce the structure of the network complexity, structural optimization of the neural network provides a new train of thought. 
The HMM-Hidden Markov Models. A hidden Markov model describes a limited set of states, one of these states can be transferred to another at a certain probability, at the same time when the shift output, output according to certain probability, and the limited number of output. For the above three questions, the first problem is to assess, can be used to determine the best model. The second problem is that the decoding problem, can be used to find the most likely to generate the observed sequence of states. The third issue is training, and can be used to estimate the parameters as of the model from the existing data. In the Fig. 3, we demonstrate the HMM architecture.

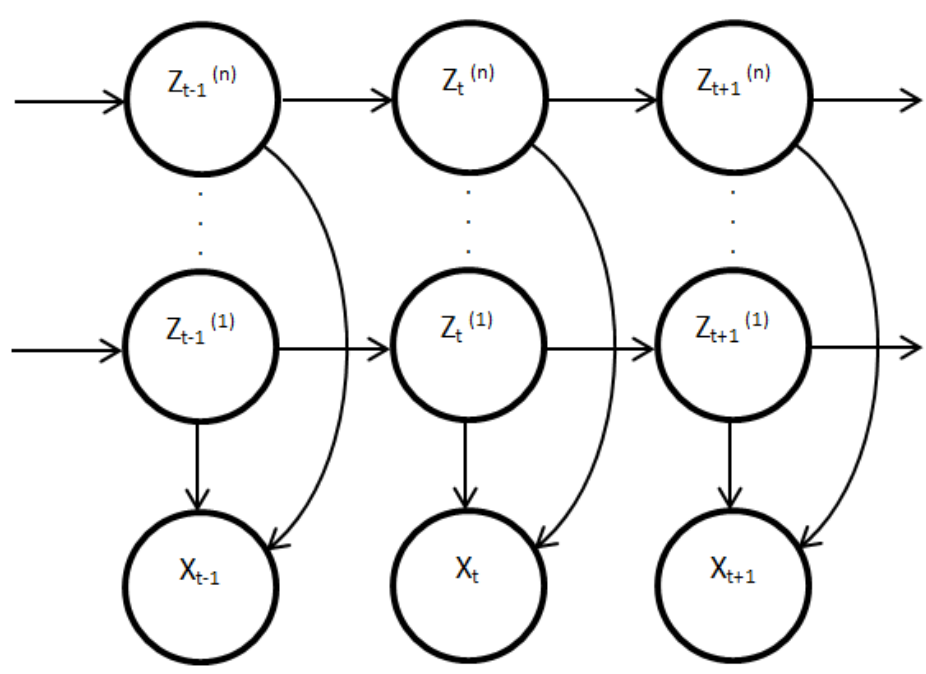

Figure 3. The HMM-Hidden Markov Model

Markov chain is used to generate training samples with the samples of the progressive distribution characteristics for the construction of support vector machine model of it is not important, the sample converge to the optimal sampling density function of velocity is the key [8].

If only using Markov chain state points as the training sample, namely only take sample points of failure domain, do so on the one hand makes, support vector machine (SVM) classification method cannot be used, because it requires security and failure of the two kinds of samples to training support vector machine (SVM), on the other hand, the Markov chain state point form samples of the general will appear the phenomenon of repetition, which left the state does not conform to the conditions of optional sample, and the alternative sample points are calculated the same, the value of the limit state function contains the response of the structure information, so should replace the duplicate sample points with optional sample points as the training sample, to obtain the support vector machine model.

The Enhance Algorithm for De-Noising. Improved threshold wavelet associated with the multi-dimension infrared image denoising algorithms, it is a threshold denoising is supplemented, the multi-dimension correlation denoising combining denoising algorithm, namely on the basis of the improved threshold denoising, also using the wavelet transform of the multi-dimension relevance to neighboring threshold of wavelet coefficients to estimate method of infrared image denoising.

Multiwavelet and is similar to the traditional wavelet denoising principle, namely on the basis of wavelet decomposition coefficients, setting refer to value processing wavelet coefficients, with the noise of the corresponding coefficient of zero, then using wavelet reconstruction signal. Compared with traditional wavelet and multiwavelet with symmetry, orthogonality, short support, the high order vanished moments, so based on wavelet transform denoising can not only complete the merits of the traditional wavelet denoising, more practical and more flexible.

$$
\delta=\varepsilon \times \sqrt{2 \ln (n)}
$$


In order to better protect the edge of infrared image details, need to introduce a general formula of the threshold shrinkage factor, but if the introduction of contraction factor value is too small, can lead to obtain the threshold value is too small that is expressed as the follows.

$$
\delta_{\text {revised }}=\delta \otimes \exp \left(\frac{1}{N}-1\right)
$$

Signal and noise in wavelet domain with different correlation, signal on the corresponding position between the scale of the wavelet coefficient has a strong correlation, while the wavelet coefficients of the noise has weak correlation or irrelevant. Scale correlation denoising is the use of image signal and noise in wavelet transform domain are related to the different characteristics of the noise. Although hard threshold is a natural choice, is able to keep the image edge details, but, because of the hard threshold with discontinuous function can cause ringing in the image denoising, pseudo Gibbs effect; Although relatively smooth soft threshold processing, can reduce the ringing and pseudo Gibbs effect, but due to the inherent deviation that will cause the image blur. For measuring the mentioned basic performance of the algorithm, we propose the new standard as the follows.

$$
P S N R_{\text {new }}=10 \times \lg \frac{255 \times 255}{M S E}
$$

\section{Conclusion}

In this paper, we conduct research on the new image de-noising methodology based on the neural network and basic HMM-hidden Markov models. The response surface method and artificial neural network can be regarded as regression methods as they are all through the empirical risk minimization principle to construct approximate explicit expressions of the implicit limit state function. The calculation precision of conventional response surface method are two main factors: the influence of the response surface function form and sampling point location, and the response surface method of the fitting function is given in advance. To optimize the mentioned challenges and the corresponding architecture, we integrate the neural network and basic HMM-hidden Markov models to modify the procedures of the traditional image de-noising methodology that achieves better performance.

\section{References}

[1] R. M. Yan and Y. Liu: IEEE Transactions on Image Processing, Vol. 22 (2013) No.12, p.4689.

[2] H. Om and M. Biswas: Journal of Signal and Information Processing, Vol. 3 (2012) No. 1, p.109.

[3] S. T. Li, L. Y. Fang and H. T. Yin: IEEE Transactions on Biomedical Engineering, Vol. 59 (2012) No.2, p.417.

[4] F. Adamo, G. Andria, F. Attivissimo, A. M. L. Lanzolla and M. Spadavecchia: Measurement, Vol. 46 (2013) No. 8, p.2447.

[5] J. C. D. L. Reyes and C. B. Schönlieb: Inverse Problems and Imaging, Vol. 7 (2013) No.4, p.1183.

[6] J. H. Pang, G. Cheung, A. Ortega and O. C. Au: 2015 IEEE International Conference on Acoustics, Speech and Signal Processing (South Brisbane, QLD, April 19-24, 2015), p.2294.

[7] G. C. Liu, H. Zhong and L. C. Jiao: IEEE Transactions on Image Processing, Vol. 24 (2015) No. 3, p. 862.

[8] C. Kervrann: Advances in Neural Information Processing Systems (Palais des Congrès de Montréal, Montréal CANADA, December 8-13, 2014). p. 1. 